\title{
Exploitation Des Ressources Biologiques Et Dynamique De La Foret Classee De La Mekrou Au Benin
}

\author{
Boni Sounon Bouko \\ Paulin Jésutin Dossou
}

Département de géographie et Aménagement du Territoire, Faculté des

Lettres, Arts et Sciences Humaines, Université de Parakou, Bénin.

\section{Boureima Amadou}

Faculté des Lettres et Sciences Humaines, Université ABDOU M

OUMOUNI de Niamey, Niger.

\section{Brice Sinsin}

Laboratoire d'Ecologie Appliquée, Faculté des Sciences Agronomiques, Université d'Abomey-Calavi, Bénin.

\section{doi: 10.19044/esj.2016.v12n36p228 URL:http://dx.doi.org/10.19044/esj.2016.v12n36p228}

\section{Abstract}

The dynamics study of the preserved forest of Mekrou was discussed. The methodology used includes documentary research, Phytosociological surveys, diachronic analysis of satellite imagery, socio-economic surveys of different social and professional groups and the analysis and interpretation of data. It is obvious that in the area of the study, agriculture, animal husbandry, forestry and the use of medicinal plants are the main forms of natural resources exploitation. This operation, with population growth leads to a regressive evolution of plants and natural landscapes in favor of agricultural fields and fallow. The area of natural vegetation increased from 70, 53\% in 1992 to $39,33 \%$ in 2012 in favor fields and fallow land increased from $17,88 \%$ to 48 , $77 \%$ in 2012. The numerical classification based on the presence-absence of vegetation species allowed us to individualize 6 vegetal communities. The Anogeissus leiocarpus, Khaya senegalensis and gallery forests group is the most diversified and most concentrated while Parkia biglobosa, Vitellaria paradoxa Field group is less diversified and less dense. The impact of soil occupation dynamics has resulted in the fragmentation of natural formations and reducing species diversity and density of ligneous.

Keywords: Dynamic, natural resources, human pressure, Classified Forest of Mekrou. 


\section{Résumé}

La dynamique de l'occupation du sol de la forêt classée de la Mékrou résulte de la forte demande des produits forestiers et agricoles affectant des processus socio-économiques et environnementaux variés. La démarche méthodologique utilisée comporte la recherche documentaire, les relevés phytosociologiques, l'analyse diachronique des images satellitales, les enquêtes socio-économiques auprès des différentes couches socioprofessionnelles puis l'analyse et l'interprétation des données. Dans la région, l'agriculture, l'élevage, l'exploitation forestière et le prélèvement des plantes médicinales sont les principales formes d'exploitation des ressources naturelles. Cette exploitation, conjuguée avec la croissance démographique a abouti à une évolution régressive des paysages végétaux au profit des champs et jachères. La superficie des formations végétales naturelles est passée de $70,53 \%$ en 1992 à 39,33\% en 2012 au profit des champs et jachères qui sont passée de $17,88 \%$ à $48,77 \%$ en 2012 . La classification numérique basée sur la présence-absence des espèces végétales de ces formations végétales a permis d'individualiser 6 groupements végétaux. Le groupement à Anogeissus leiocarpus et Khaya senegalensis des forêts galeries est le plus diversifié et le plus dense alors que le groupement à Parkia biglobosa et Vitellaria paradoxa des champs est le moins diversifié et le moins dense. L'impact de la dynamique d'occupation du sol s'est traduit par la fragmentation des formations végétales, la réduction de la diversité spécifique et la densité des ligneux.

Mots-clés : Dynamique, ressources végétales, pression anthropiques, Forêt Classée de la Mékrou.

\section{Introduction}

Les forêts en Afrique constituent d'importante source d'emplois, d'énergie, d'aliments nutritifs et d'une gamme variée de biens, ainsi que des services écosystémiques (FAO, 2010). Elles constituent les réserves de biens et services, représentent l'un des grands réservoirs en diversité génétiques et, jouent un rôle fondamental dans la satisfaction des nombreux besoins alimentaires, énergétique, médicinaux et culturels des populations locales (Ouédraogo, 2006).

Cependant, l'exploitation des ressources forestières produit en Afrique une perte annuelle de 3,414 millions d'hectares de superficie soit $0,49 \%$ (FAO, 2010). Cette situation est aussi perceptible en Afrique de l'Ouest qui enregistre un taux annuel de régression $(0,46 \%$ par an) des formations forestières (FAO, 2010). 
Le Bénin dont le taux de couverture n'est que de $0,40 \%$, a un taux annuel de déforestation de 1,06\% (FAO, 2010). Aujourd'hui, la végétation du domaine soudanien du Bénin présente une évolution régressive sous l'effet conjugué des feux de végétation, des cultures et de l'élevage (Oumorou ,1998). Il est loisible de reconnaître que les activités économiques ont un impact négatif sur le milieu (Le barbe et al., 1993)

La forêt classée de Mékrou n’est pas épargnée de cette situation. Elle est devenue la cible privilégiée des exploitants de bois, des agriculteurs et des éleveurs. Sous la pression et la croissance démographique conjuguée à une paupérisation grandissante, les populations riveraines violent ce domaine protégé à la recherche de nouvelles terres cultivable ou des coupes pour le bois de feu. Ces activités contribuent à la dégradation des écosystèmes de cette aire protégée.

L'objectif visé par cette étude est de prendre en compte les différentes modifications qu'a subies la forêt classée de la Mékrou en mettant un accent particulier sur l'exploitation des ressources biologiques qui ont été à la base de cette évolution. De manière spécifique, il s'agit :

milieu ;

- d'analyser les différents types d'activités et leurs impacts sur le

- de cartographier à l'aide des images satellites, les changements subis par les paysages végétaux dans le temps ce qui permettra d'apprécier objectivement la tendance évolutive des formations végétales et de dégager les effets de ces changements sur la structure et la diversité spécifique de la végétation.

\section{Matériel et méthodes \\ Milieu d'étude}

Créée en 1950, la forêt classée de la Mékrou (figure 1), d'une superficie totale de 9680 hectares, est administrativement à cheval sur les Communes de Kouandé et de Péhunco. Limitée au Nord-Est par le village de Doh, au Sud par les localités de Makrougourou et de Gbéda à travers l'axe Kouande-Péhunco et à l'Ouest par la ville de Kouandé, la forêt classée de la Mékrou est située entre $10^{\circ} 14^{\prime} 55^{\prime}$ ' et $10^{\circ} 23^{\prime} 3^{\prime}$ ', latitude Nord et entre $1^{\circ}$ 48 '21' ' et $1^{\circ} 54$ ' 15 ', longitude Est.

Du point de vue climatique, on observe au cours de l'année deux saisons nettement contrastées, l'une sèche de sept mois environ et l'autre humide de cinq mois, qui dure en moyenne de juin à octobre. Les précipitations moyennes annuelles sont de l'ordre de $1200 \mathrm{~mm}$. Le milieu est entièrement soumise au climat tropical de type soudano-guinéen avec une température moyenne de $27^{\circ} \mathrm{C}$. Les sols ferrugineux tropicaux sont très fréquents dans la Forêt Classée tandis que les autres types de sols sont très faiblement représentés (sols hydromorphes, sols ferrallitiques). La végétation 
est dominée par des savanes parsemées d'îlots de forêts claires et de forêts galeries. Le tapis herbacé, consistant, est essentiellement composé de graminées.

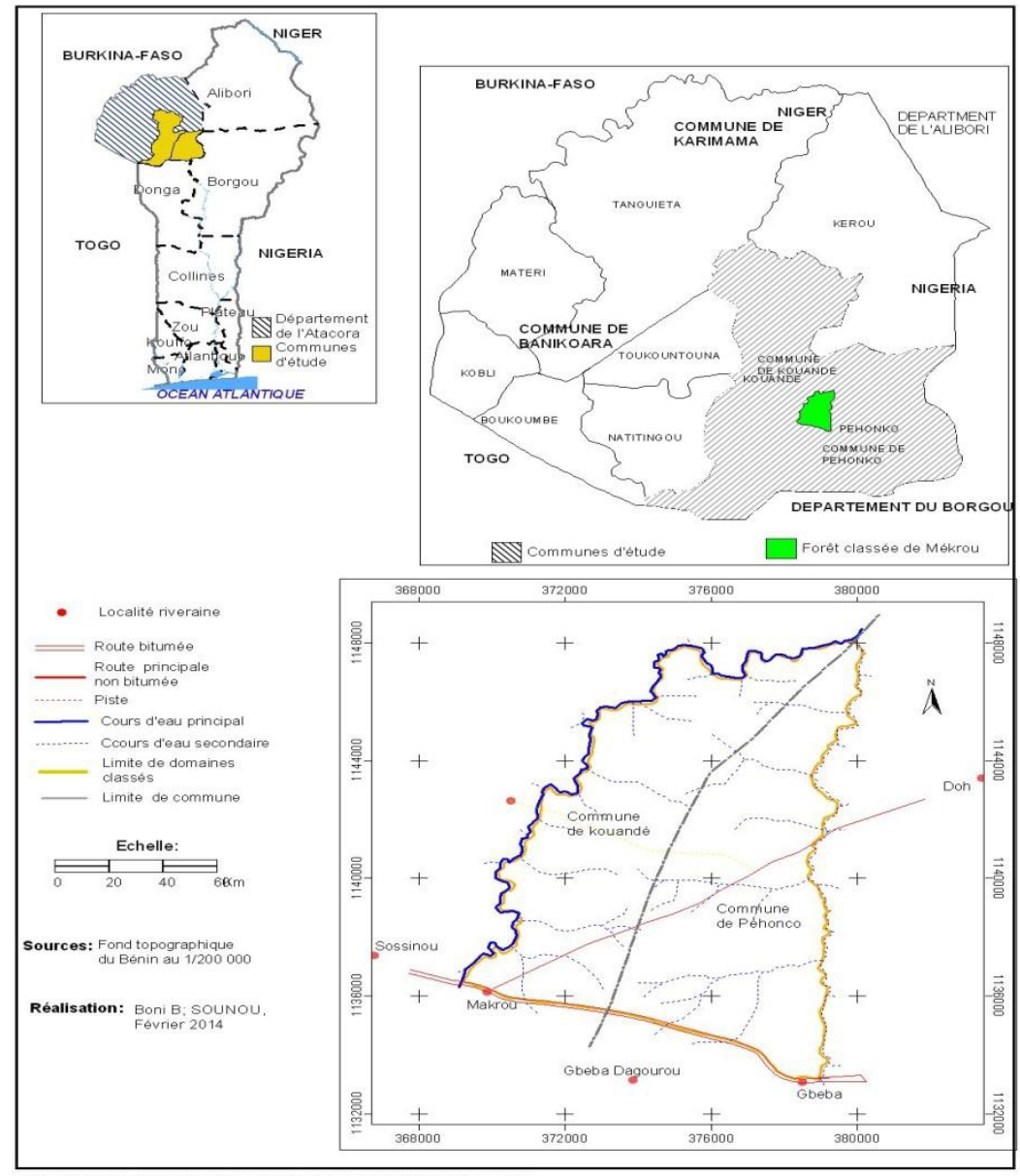

Figure 1: Situation géographique de la Forêt classée de Mékrou

\section{Méthodes}

\section{Collecte des données}

\section{Matériels}

Le matériel utilisé dans notre étude est composé : des photos aériennes panchromatiques N/B mission IGB Burkina, octobre 1982 et Ortho image GED-EYA1, juin 2012; le Logiciel Arc GIS 9.3 pour les travaux de cartographie et analyses. 


\section{Inventaire floristique}

Les relevés phytosociologiques ont été effectués suivant la méthode sigmatiste de Braun-Blanquet (1932). La caractérisation de la végétation de l'aire d'étude a été réalisée au moyen d'un inventaire forestier. Une stratification de la végétation a été effectuée en tenant compte du type de formation végétale. Ainsi, 49 placeaux carrés de $30 \mathrm{~m}$ de côté chacun ont été installés de façon aléatoire en forêt claire, savane arborée, champ et jachère.

La collecte des données dans les placeaux d'inventaire s'est faite au moyen d'un ruban pi pour la mesure des diamètres des arbres à hauteur de poitrine d'homme, à 1,30 m du sol, d'un GPS pour l'enregistrement des coordonnées géographiques des placeaux d'inventaire. Une fiche a été utilisée pour relever les différentes mensurations. Les donnée dendrométriques ont concerné le diamètre des ligneux de dbh supérieur ou égal à $10 \mathrm{~cm}$.

\section{Enquêtes socio-économiques}

L'analyse de la relation entre les populations riveraines et la forêt classée d'une part, et entre les populations et les agents forestiers d'autre part a été faite par une enquête auprès desdit acteurs. Elle a été effectuée sous forme d'entretien semi-structuré. Ces entretiens reposaient sur un questionnaire testé au préalable. Deux types de questionnaire ont été élaborés à cet effet et administrés respectivement aux populations locales et aux gestionnaires de Forêt Classée de la Mékrou. Les données recueillies concernaient entre autres les activités pratiquées à l'intérieur et à la périphérie de la forêt et la perception des populations riveraines de la réserve.

A cet effet, quatre villages riverains ont été prospectés et 101 personnes (agriculteurs, exploitants forestiers, charbonniers, forestiers et éleveurs) ont été enquêtées. Les informations ont été complétées par des observations directes dans les champs et dans la forêt avec l'aide des agents forestiers et des guides locaux. Cette prospection a permis d'identifier les principales activités illicites menées dans la forêt classée et de recenser les différentes espèces ligneuses exploitées. Les observations ont été consignées dans un carnet de notes.

\section{Traitement des données}

\section{Calcul du rythme d'évolution des unités d'occupation du sol}

Les superficies des différentes unités d'occupation du sol ont permis de quantifier les tendances évolutives ainsi que le taux moyen d'expansion spatiale annuel des formations végétales.

Soit S1, la superficie d'une formation végétale en1992;

Soit S2, la superficie de la même formation végétale en 2012 ;

Soit $\beta S$, la variation de la superficie de ladite formation végétation entre 1992 et 2012 


$$
\beta \mathrm{S}=\mathrm{S} 2-\mathrm{S} 1
$$

Pour une formation végétale, on peut alors assister à l'un des trois cas suivants :

- $\quad \beta S=0$, alors il y a stabilité

- $\quad \beta S>0$ alors il y a évolution progressive

- $\quad \beta S<0$, alors il y a évolution régressive

Le Taux d'évolution globale $(\mathrm{Tg})$ a été évalué à partir de la formule suivante :

$\mathrm{Tg}=\beta \mathrm{S} / \mathrm{S} 1 \mathrm{x} 100$

\section{Evaluation de la diversité floristique}

\section{Classification hiérarchique des relevés}

La matrice de 49 relevés et 82 espèces végétales recensées est soumise à une analyse sur la base de présence-absence des espèces par relevé à l'aide du Logiciel PC ORD 5 sous Detranted Canonial Analysis (DCA). Des groupements végétaux ont été obtenus.

\section{Calcul des paramètres structuraux et de diversité}

Pour chaque groupement végétal identifié, les paramètres de diversité floristique que sont la richesse spécifique, l'indice de diversité de Shannon et l'équitabilité de Pielou ont été calculés. De même, pour chaque groupement végétal identifié, les paramètres dendrométriques que sont la densité d'arbres et la surface terrière ont été calculés.

Richesse spécifique ( $R$ en espèces): elle représente le nombre total d'espèces présentes dans un milieu donné.

Indice de diversité de Shannon ( $H$ en bits), défini par la formule suivante :

$$
\mathbf{H}=-\sum_{i=1}^{n}\left(\frac{N i}{N}\right) \log 2\left(\frac{N i}{N}\right)
$$

$n_{i}=$ nombre d'individus de l'espèce $i$ et $n=$ nombre total d'individus inventoriés dans les placeaux.

Equitabilité de Pielou (Eq): elle est une mesure de la stabilité du peuplement et équivaut au rapport de $H$ à l'indice maximal théorique dans le peuplement $\left(H_{\max }\right)$ :

$$
\mathbf{E Q}=\mathbf{H} / \mathrm{H}_{\max }
$$

Surface terrière ( $G$ en $\mathrm{m}^{2} / \mathrm{ha}$ ): pour tout le peuplement, elle est la somme de la section transversale à hauteur d'homme $(1,30 \mathrm{~m})$ de tous les arbres de $\mathrm{dbh} \geq 10 \mathrm{~cm}$, à l'intérieur du placeau.

$$
\mathbf{G}=\frac{\pi}{40000 s} \sum_{\mathbf{i}=1}^{\mathbf{n}} \mathbf{d i}^{2}
$$


Avec di le diamètre (en $\mathrm{cm}$ ) de l'arbre $i$ du placeau considéré et $\mathrm{s}$, la surface du placeau en ha.

Densité du peuplement ( $N$ en arbres/ha): elle est le nombre moyen d'arbres de $\mathrm{dbh} \geq 5 \mathrm{~cm}$ par hectare. Elle est donnée par la formule:

$$
\mathbf{D}=\mathrm{N} / \mathrm{s}
$$

$N$ est le nombre total d'arbres par placeau et $s$, la surface du placeau en ha.

\section{Données socio-économiques}

Le traitement des données d'enquête collectées sur le terrain s'est effectué manuellement à l'aide d'une grille de dépouillement. Ce dépouillement a permis d'apprécier la fréquentation de la forêt classée par les populations et les impacts de leurs activités.

\section{Résultats}

\section{Les formes d'exploitation des ressources biologiques}

Il est a noté que plusieurs formes d'exploitation des ressources biologiques ont été identifiées. Au nombre de ces formes d'exploitation on a : l'agriculture, l'élevage, l'exploitation forestière, la carbonisation, le prélèvement de plantes médicinales, la transhumance et la chasse.

\section{L'agriculture}

La mise en culture des terres de la réserve concerne principalement sa zone périphérique. Des informations collectées sur le terrain $(90 \%$ des enquêtés), le besoin en terre cultivable suite à l'augmentation de la population, pousse les agriculteurs à s'infiltrer à l'intérieur de la forêt. Les espèces cultivées sont l'igname, le maïs, le sorgho et le coton. La création de nouveaux champs correspond à des soucis de satisfaction de certains besoins. L'exploitation agricole repose essentiellement sur le capital foncier. Donc, il va de soi que les producteurs soient guidés dans la mesure du possible par l'accroissement des superficies. L'impact environnemental qui est perceptible dans la zone d'étude est sans conteste la déforestation consécutive à l'extension des superficies cultivées. Elle constitue une menace pour certaines essences ligneuses et non ligneuses. En effet, dans les champs cotonniers, les défriches (sélectives ou non) sont effectuées et en général il ne reste que quelques espèces ligneuses (Vitellaria paradoxa) ; ces dernières sont aussi en nombre réduit (photo 1 et 2). 


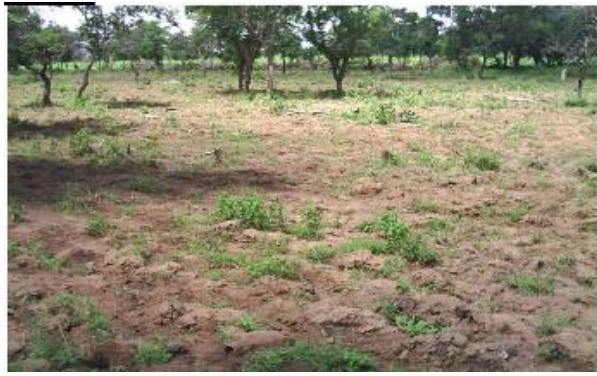

Photo $\mathrm{N}^{\circ} 1$ : Parcelle déboisée

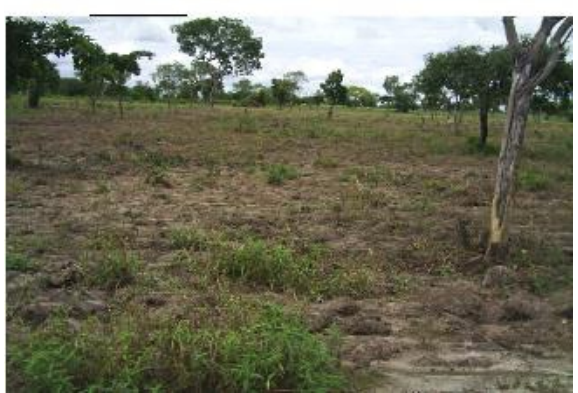

Photo $\mathbf{N}^{\circ} 2$ : Parcelle d'exploitation défrichée Source : prise de vue, les auteurs, 2016.

\section{L'exploitation forestière}

Le bois de chauffe : Le bois de feu et ses dérivées constituent la principale source d'énergie domestique utilisée par la quasi-totalité de la population rurale comme urbaine (environnante voire éloignée), pour la cuisson d'aliments, l'artisanat, ou á diverses autres fins. Dans le secteur d'étude, la plupart des populations situées aux abords de la piste principale s'adonnent à l'exploitation et à la commercialisation du bois de feu. La collecte du bois se fait soit par coupe de rejets verts qui sont ensuite séchés, soit par détachements de branches sur arbres secs (morts sur pieds), ou par abattage systématique de gros arbres qui sont ensuite fendus. Cette collette se réalise au moyen d'outils rudimentaires tels que les machettes, les cognées ou les feux. D'après les enquêtes, les espèces les plus appréciés comme bois de feu sont Anogeissus leiocarpus (78,86 \%), Prosopis africana (71,25\%), Pterocarpus erinaceus (57,81 \%), Terminalia macroptera (39,15\%), Gardenia ternifolia $(13,51 \%)$, etc. parce qu'elles sèchent très vite, conservent la flamme, prennent feu rapidement et ne font pas trop de fumée.

La carbonisation : La carbonisation, ou production de charbon de bois n'est assurée que par un nombre limité d'exploitants pour qui cette activité est généralement secondaire. La meule de carbonisation est située à l'intérieur de la réserve (Planche. Les espèces utilisées dans la fabrication du charbon de bois sont: Prosopis africana (82,05\%), Anogeisus leiocarpus (75,22 \%), Pterocarpus erinaceus $(60,17 \%)$, Terminalia spp. (26,79 \%), Khaya senegalensis $(11,32 \%)$.

La recherche du bois d'œuvre: l'exploitation du bois d'œuvre constitue une activité qui décime les ressources ligneuses. Dans le milieu d'étude, la production locale du bois d'œuvre pour le commerce est difficile à contrôler en raison du fait que l'abattage et le sciage se font souvent de façon illicite et à petite échelle. Malgré la présence des forestiers, le phénomène d'abattage du bois d'œuvre se poursuit pour les espèces suivantes : Afzelia africana, Pterocarpus erinaceus, Isoberlinia doka et Khaya senegalensis. Les enquêtes auprès des populations locales ont permis d'établir que les essences 
de valeur commerciale (bois d'œuvre, bois de service, etc.) ont été systématiquement exploitées, non seulement pour les besoins propres mais aussi pour des raisons économiques compte tenu de la demande urbaine en bois d'œuvre mais aussi une grande partie est exportée. L'inefficacité du contrôle de l'administration forestière a largement contribué à cette exploitation sélective des essences de valeur. D'après les responsables de l'administration forestière et les petits exploitants forestiers interrogés, seuls quelques massifs classés et les domaines protégés de l'Etat constituent actuellement les zones refuges où l'on pourrait encore rencontrer des arbres à valeur commerciale.

\section{L'élevage transhumant}

La transhumance à l'intérieur de la forêt classée, des troupeaux de bovins en provenance de l'Est du pays et des pays sahéliens est un phénomène généralisé. L'effectif du cheptel ne cesse d'augmenter chaque année et aucun endroit de la réserve n'est épargné. Cette situation pourrait s'expliquer par la bonne maîtrise des différentes pathologies animales.

La disponibilité du fourrage avec des points d'eau permanents dans la forêt classée qui constituent autant de points d'attraction pour les animaux domestiques.

Le cheptel présent doit représenter quelques centaines de têtes. Tous les pâturages, les abords de mares, les points d'eau, montrent des traces nombreuses de passage de ces animaux. Au même moment, la nécessité de compléter surtout en période de saison sèche, l'alimentation ordinaire des animaux par le pâturage aérien, amène les éleveurs à se livrer tous azimuts à l'émondage souvent exagéré des essences fourragères des aires protégées. Au voisinage des nombreux campements d'éleveurs, les espèces végétales émondées pour la nourriture des troupeaux témoignent de l'impact négatif de la pression pastorale sur les ressources végétales de la forêt classée. Les espèces végétales consommées par le bétail sont: Afzelia africana (menacée d'extinction dans la zone à cause de sa fréquence d'utilisation), Prosopis africana, Pterocarpus erinaceus, Khaya senegalensis, Stereospermum kunthianum.

En somme, l'agriculture, l'élevage, l'exploitation forestière et l'utilisation des espèces végétale dans la médecine traditionnelle ont imprimé une certaine physionomie à la forêt classée de la Mékrou. Cette physionomie est mieux appréciée à travers les différents supports cartographiques réalisés.

\section{Dynamique de l'occupation du sol entre 1992 et 2012}

La dynamique des formations végétales de la forêt classée de la Mékrou est appréciée par l'utilisation de plusieurs trames caractéristiques de la progression, de la stabilité et de la régression. Le tableau 1 et les figures 2 
et 3 illustrent les différents changements opérés au niveau des unités d'occupation du sol. L'examen de la situation des différentes unités d'occupation du sol en 2012 par rapport à l'année de référence (1992) montre une forte extension des champs et jachères $(30,89 \%)$ suivi des zones habitées qui ont connu une augmentation sensible.

Tableau 1: Variation des superficies de 1992 à 2012

\begin{tabular}{|c|r|r|r|r|r|r|r|}
\hline $\begin{array}{c}\text { Formation } \\
\text { végétation }\end{array}$ & $\begin{array}{c}\text { Superficie } \\
1992 \text { en } \\
\text { Ha }\end{array}$ & Pourcentage & $\begin{array}{c}\text { Superficie } \\
2012 \text { en } \\
\text { Ha }\end{array}$ & Pourcentage & \multicolumn{1}{|c|}{$\begin{array}{c}\text { Différence } \\
\text { entre } \\
1992 \text { et }\end{array}$} & Pourcentage & Observation \\
\hline Agglomération & 18 & 0,19 & 48 & 0,50 & 30 & 0,31 & Progression \\
\hline $\begin{array}{c}\text { Forêt claire } \\
\text { Savane boisée }\end{array}$ & 2027 & 20,94 & 1027 & 10,61 & -1000 & $-10,33$ & Regression \\
\hline $\begin{array}{c}\text { Mosaïque de } \\
\text { Culture et } \\
\text { jachère }\end{array}$ & 1731 & 17,88 & 4721 & 48,77 & 2990 & 30,89 & Progression \\
\hline Forêt galerie & 578 & 5,97 & 458 & 4,73 & -120 & $-1,24$ & Regression \\
\hline Plantation & 1103 & 11,39 & 1103 & 11,39 & 0 & 0 & Stabilité \\
\hline Plan d'eau & 1 & 0,01 & 1 & 0,01 & 0 & 0 & Stabilité \\
\hline $\begin{array}{c}\text { Savane } \\
\text { arborée } \\
\text { arbustive }\end{array}$ & 4222 & 43,62 & 2322 & 23,99 & -1900 & $-19,63$ & Regression \\
\hline Total & $\mathbf{9 6 8 0}$ & 100 & $\mathbf{9 6 8 0}$ & 100 & & & \\
\hline
\end{tabular}

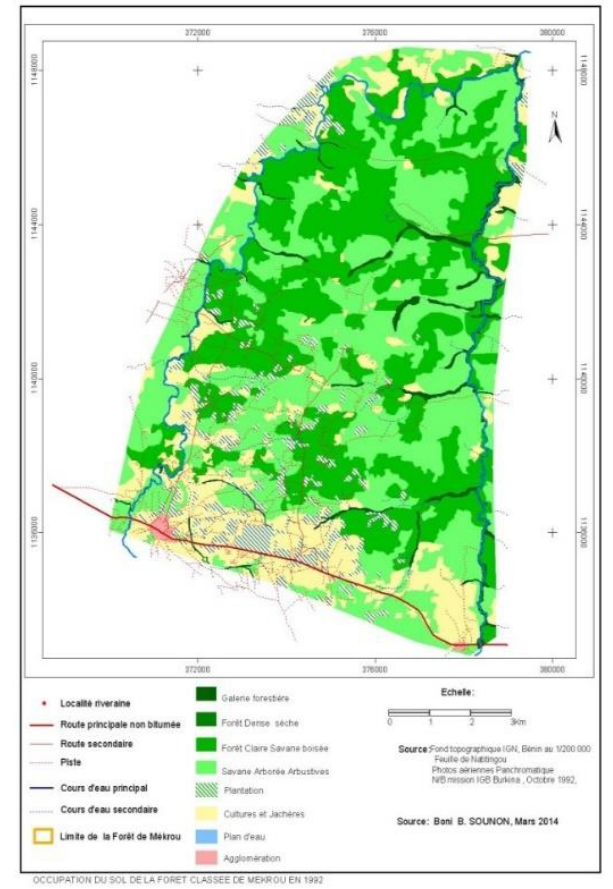

Figure 2 : Occupation du sol en 1992

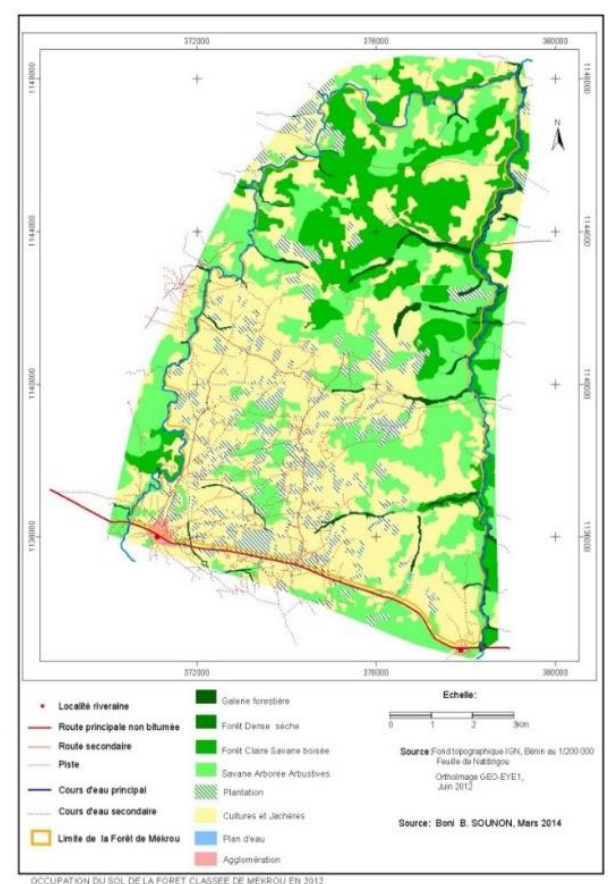

Figure 3 : Occupation du sol en 2012

Ces résultats mettent en lumière l'emprise spatiale des activités de production agricole et concordent avec l'augmentation des besoins de facteurs de production supplémentaire (nouvelles terres agricoles) pour faire face à la 
croissance démographique galopante de la zone. Il s'en suit d'importantes modifications dans la couverture végétale qui s'opèrent principalement au détriment des zones boisées. Ainsi, au cours des deux dernières décennies, les savanes arborées et arbustives de la zone d'étude ont perdu environ le 1/5 de leur superficie initiale $(-19,63 \%)$. La même tendance à la réduction spatiale a été observée au niveau des forêts claires et des savanes boisées $(-10,33 \%)$ et dans une moindre mesure, au niveau des forêts galeries $(-1,24 \%)$. Corrélativement à la réduction des strates boisées, on note une tendance à l'extension des mosaïques de culture et de jachère. Occupant une superficie de 1731 ha en 1992, elles ont atteint 4721 ha en 2012, ce qui correspond en valeur absolue à une augmentation de 2990 ha entre ces deux dates, soit une croissance de $30,89 \%$. Les plans d'eau et les plantations sont par contre restés stables.

\section{Individualisation des groupements végétaux}

L'inventaire au sein des 49 placeaux étudiés a permis d'obtenir 58 espèces constituées uniquement des espèces ligneuses réparties en 51 genres et 27 familles. Ces relevés ont été soumis à une classification hiérarchique. Le diagramme issu de cette analyse a permis d'identifier 6 groupements végétaux. La figure 4 présente le dendrogramme des relevés phytosociologiques obtenus.

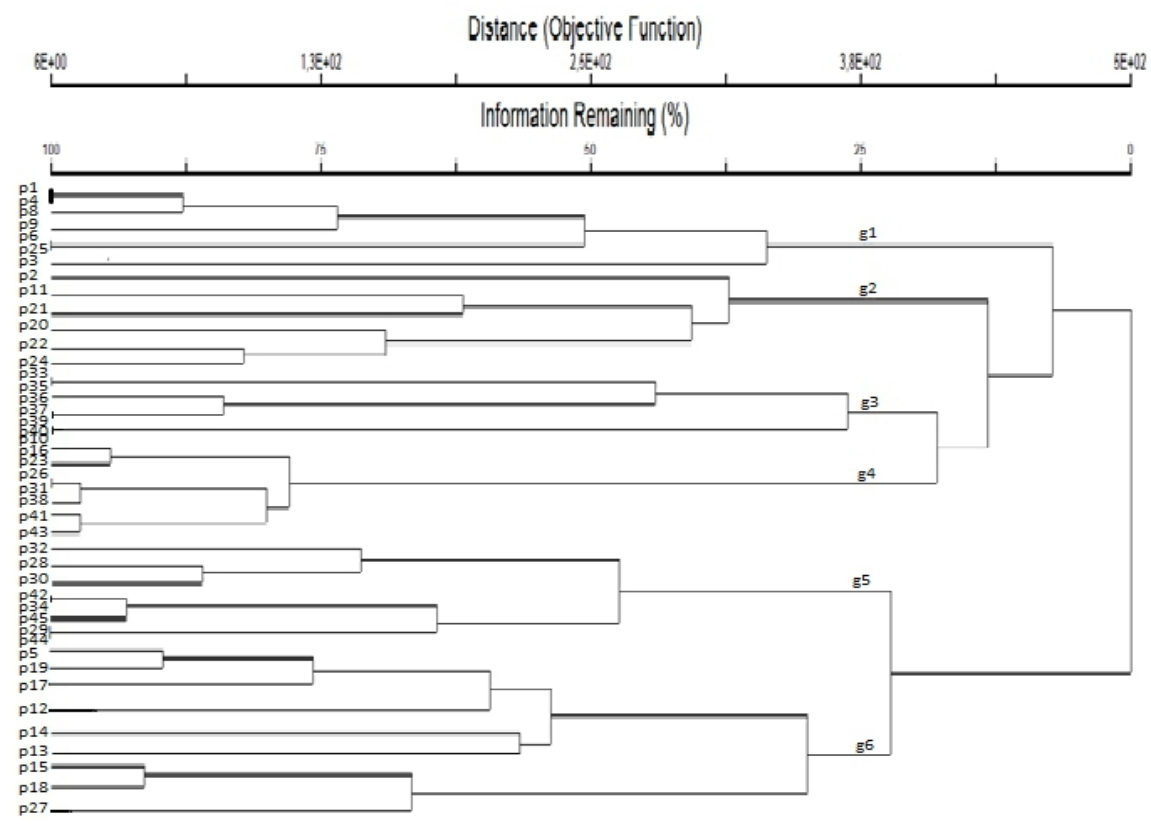

Figure 4 : Dendrogramme des relevés phytosociologiques des groupements végétaux 


\section{Description des groupements végétaux}

La structure diamétrique des peuplements ligneux des divers groupements végétaux recensés dans la forêt classée de la Mékrou se présente comme l'indiquent les figures $5,6,7,8,9$, et 10 .

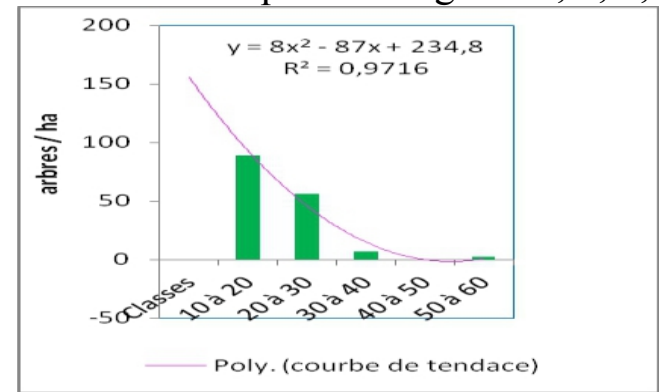

Figure 5 : Structure diamétrique du G1

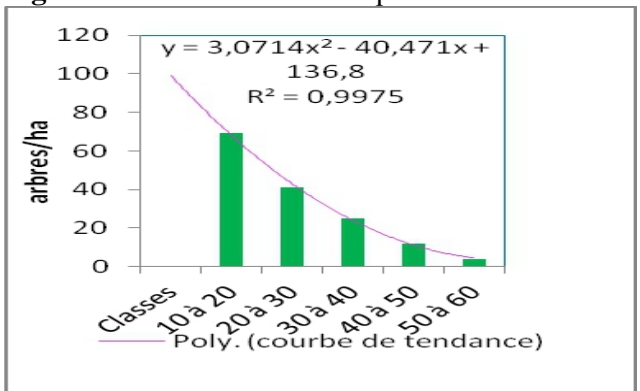

Figure 7 : Structure diamétrique du G3

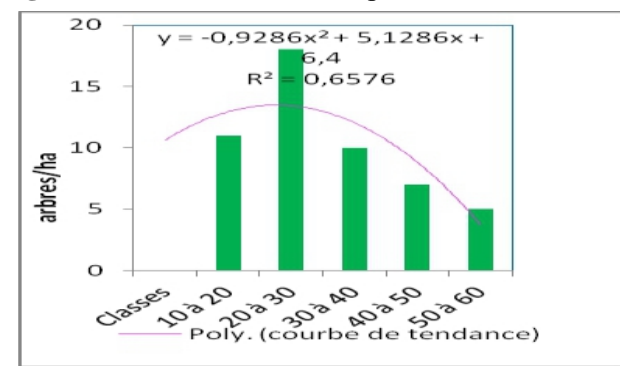

Figure 9 : Structure diamétrique du G5

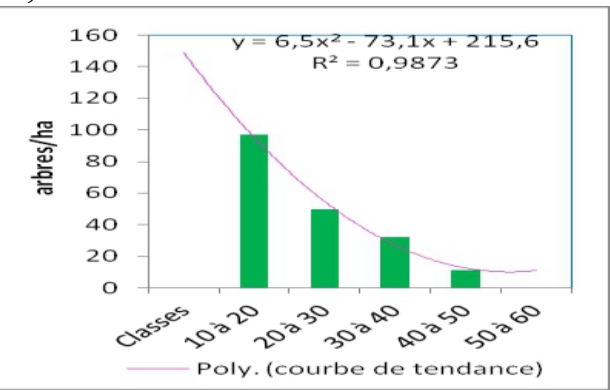

Figure 6 : Structure diamétrique du G2

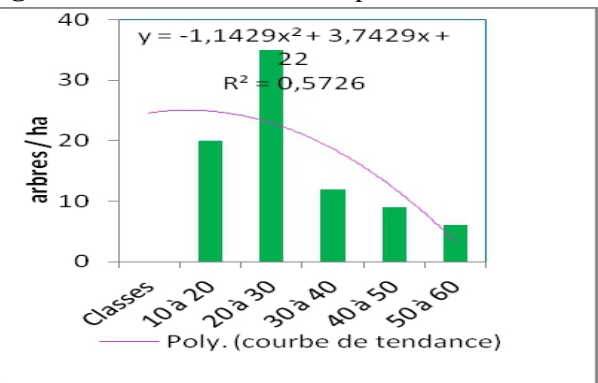

Figue 8 : Structure diamétrique du G4

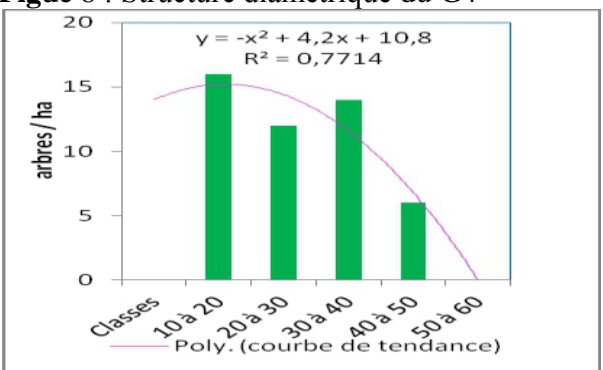

Figure 10 : Structure diamétrique du G6

Le groupement G1 composé de 7 relevés est le groupement à Anogeissus leiocarpus et Khaya senegalensis des forêts galeries. Les espèces caractéristiques de ce groupement sont: Khaya senegalensis, Anogeissus leiocarpus, Daniellia oliveri. Les valeurs moyennes de l'indice de diversité de Shannon et de l'équitabilité de Pielou sont respectivement de 4,40 $\pm 0,59$ bits et de $0,88 \pm 0,1$. La composition floristique de ce groupement est alors très diversifiée. La surface terrière moyenne est de 14,05 $\pm 5,68 \mathrm{~m}^{2} / \mathrm{ha}$. La densité des ligneux de $\mathrm{dbh}>10 \mathrm{~cm}$ est de $380 \pm 132$ individus/ha. La structure diamétrique de ce groupement est ajustée à une fonction polynomiale ayant une structure en $\mathrm{J}$ renversé confirmant la prédominance des individus de petits diamètres (Figure 5). 
Le groupement $\mathrm{G} 2$ constitué de 6 relevés est le groupement à Isoberlinia doka et Daniellia oliveri des forêts claires. Les espèces caractéristiques du groupement sont: Isoberlinia doka, Daniellia oliveri, Acassia sieberiana. Les valeurs moyennes de l'indice de diversité de Shannon et de l'équitabilité de Pielou sont respectivement de 3,51 $\pm 0,73$ bits et de 0,75 $\pm 0,19$. L'indice de diversité de Shannon élevé montre la grande diversité des espèces végétales de ce groupement. La surface terrière moyenne est de 10,32 $\pm 6,51 \mathrm{~m}^{2} /$ ha. La densité des ligneux de $\mathrm{dbh}>10 \mathrm{~cm}$ est de $186 \pm 162$ individus/ha. La structure par classe de diamètre de ce groupement est ajustée à une fonction polynomiale qui montre une décroissance rapide des individus avec l'augmentation des diamètres (Figure 6).

Le groupement G3 formé de 6 relevés est le groupement à Parkia biglobosa et Vitellaria paradoxa des champs. Les espèces caractéristiques sont: Vittelaria paradoxa et Parkia biglobosa. Les valeurs moyennes de l'indice de diversité de Shannon et de l'équitabilité de Pielou sont respectivement de $2,53 \pm 0,72$ bits et de $0,66 \pm 0,07$. La surface terrière moyenne est de $3,39 \pm 8,68 \mathrm{~m}^{2} / \mathrm{ha}$. La densité des ligneux de $\mathrm{dbh}>10 \mathrm{~cm}$ est de $34 \pm 112$ individus/ha. Ces valeurs de densité de ligneux et de la surface terrière indiquent clairement que le milieu est dégradé. La structure diamétrique de ce groupement qui s'ajuste mieux à une fonction polynomiale (Figure 7). On remarque une décroissance des effectifs au fur et à mesure que les diamètres augmentent.

Le groupement G4 composé de 7 relevés est le groupement à Isoberlinia doka et Vitellaria paradoxa des forêts claires, des champs et des jachères. Les espèces caractéristiques de ce groupement sont: Isoberlinia doka, Isoberlinia tomentosa, Daniellia oliveri et Anogeissus leiocarpus. Les valeurs moyennes de l'indice de diversité de Shannon et de l'équitabilité de Pielou sont respectivement de 4,34 $\pm 0,56$ bits et de 0,87 $\pm 0,7$. La surface terrière moyenne est de $10,50 \pm 6,38 \mathrm{~m}^{2} /$ ha. La densité des ligneux de $\mathrm{dbh}>$ $10 \mathrm{~cm}$ est de $237 \pm 122$ individus/ha. La répartition des individus par classe de diamètre présente une structure en cloche (Figure 8 ).

Le groupement G5 constitué de 8 relevés est le groupement à Daniellia oliveri, Isoberlinia doka et vitellaria paradoxa des savanes arbustives et jachères. Les espèces caractéristiques de ce groupement sont: Vittelaria paradoxa, Acassia sieberiana et Pteleopsis suberosa. Les valeurs moyennes de l'indice de diversité de Shannon et de l'équitabilité de Pielou sont respectivement de 3,14 $\pm 0,59$ bits et de 0,87 $\pm 0,9$. La composition floristique de ce groupement est alors relativement diversifiée. La surface terrière moyenne est de 5,55 $\pm 5,68 \mathrm{~m}^{2} /$ ha. La densité des ligneux de $\mathrm{dbh}>10 \mathrm{~cm}$ est de $107 \pm 132$ individus/ha. . La répartition des individus par classe de diamètre présente une structure en cloche (Figure 9). 
Le groupement G6 composé de 8 relevés est le groupement à Daniellia oliveri et Isoberlinia doka des savanes arborées et arbustives. Les espèces caractéristiques de ce groupement sont: Daniellia oliveri, Isoberlinia doka et Parinari curatelifolia. Les valeurs moyennes de l'indice de diversité de Shannon et de l'équitabilité de Pielou sont respectivement de 3,50 $\pm 0,51$ bits et de $0,78 \pm 0,9$. La surface terrière moyenne est de $6,05 \pm 0,83 \mathrm{~m}^{2} / \mathrm{ha}$. La densité des ligneux de $\mathrm{dbh}>10 \mathrm{~cm}$ est de $380 \pm 132$ individus/ha. La structure diamétrique de ce groupement est ajustée à une fonction polynomiale ayant une structure en cloche (Figure 10).

\section{Discussion}

Des facteurs locaux et régionaux peuvent expliquer l'état de dégradation actuel des formations végétales dans les aires classées en général (Houndagba et al., 2007). A l'échelle locale, ce sont les activités agricoles et l'exploitation forestière. $\mathrm{Au}$ plan régional, ce sont les modifications climatiques, notamment la tendance à la baisse des totaux pluviométriques depuis la sècheresse des années 1970 et dont les effets en termes de mouvements de populations et du bétail sont bien connus.

En effet, la forêt classée de la Mékrou est soumise à diverses formes de pression. Étant donné que la plupart des terres fertiles sont localisées à l'intérieur de la forêt, elles sont de ce fait convoitées par les agriculteurs et font ainsi l'objet de défrichements agricoles. Outre l'envahissement agricole, d'autres menaces pèsent sur la biodiversité mise en protection : la transhumance, les coupes de bois, etc. Selon Benoit (1998) in Convers et al. (2007), l'impact de la transhumance sur les ressources naturelles est essentiellement lié à la crise environnementale qui sévit dans la région ouestafricaine depuis les épisodes de grave sécheresse des années 1970 et qui se perpétue depuis avec l'augmentation de la densité démographique, en présence de populations dont le mode de vie a toujours largement reposé sur le libre accès aux ressources naturelles et à leur exploitation. Les formations naturelles sont ainsi de plus en plus fragmentées en îlots enrobés dans les paysages agraires (Hansen et al. 2000). Dans la forêt classée de la Mékrou, les conflits entre forestiers et éleveurs sont fréquents à cause de la présence de troupeaux illégaux dont les impacts sur ses écosystèmes peuvent s'avérer préjudiciables. Les travaux de Sarr (2010) dans le parc national des "deux balé" au Burkina Faso ont montré que la circulation du bétail crée des pistes et des zones de piétinement excessif partout, notamment autour des mares et dans les prairies attenantes. Ces différentes activités qui étaient autrefois interdites dans ladite réserve sont aujourd'hui menées par les populations riveraines à cause de l'augmentation de la population et de la paupérisation croissante. Les produits prélevés sont destinés aussi bien à l'utilisation domestique qu'à la vente. Ces activités ont pour conséquences la raréfaction 
de certaines espèces végétales, la diminution de la couverture forestière, la perte d'habitats des animaux et de biodiversité, l'érosion des sols le changement des régimes hydrauliques, la perturbation climatique, etc.

Sur le plan floristique, les fortes pressions anthropiques ont induit la raréfaction voir la disparition de certaines espèces. Certains auteurs ont abouti à des résultats similaires. En effet, les facteurs anthropiques perturbent fortement le fonctionnement des écosystèmes de ladite réserve engendrant ainsi la modification de la composition floristique et de la structure de la végétation (Sinsin, 1994, 2001; Hahn-Hadjali, 1998 ; Fournier et al., 2002).

La dynamique spatiale de l'espace étudié peut être appréciée en comparant l'état 1992 pris comme référence et l'état 2012. La régression des formations naturelles est de l'ordre d'environ 31,2\% en 20 ans (1992-2012) dans la Forêt Classée de la Mékrou. Ce taux est largement inférieur de ceux signalés par d'autres auteurs qui ont effectué des études sur des milieux protégés : 41\% pour l'Alibori Supérieur en 23 ans entre 1975 et 1998 (Arouna, 2002) et 45\% pour la zone cynégétique de la Djona en 22 ans entre 1975 et 1997 (Saliou, 2001) mais légèrement inférieur à celui trouvé par Adéyèmi (2002) pour la forêt classée de Wari-Maro qui est de l'ordre de $28 \%$. Le peuplement des espèces les plus recherchées comme combustibles ou bois d'œuvre à savoir Khaya senegalensis, Anogeissus leiocarpus, Afzelia africana, Burkea african et Prosopis africana, deviennent aujourd'hui moins nombreux et les grands semenciers de plus en plus rares. Ces résultats sont comparables à ceux de Akoègninou et Akpagana (1997), qui ont signalé que les jeunes pieds de ces espèces n'atteignent pas l'âge adulte avant d'être coupés pour leurs besoins en combustible.

\section{Conclusion}

L'étude diachronique fondée sur les techniques de télédétection couplées avec les investigations sur le terrain a révélé le rythme de dégradation des ressources naturelles, notamment des formations forestières, et les facteurs qui y ont concouru ou y concourent encore.

La tendance générale de la dynamique, est la régression des formations naturelles au profit des formations anthropiques. En effet, ce sont des facteurs locaux qui motivent ces changements spatio-temporels des formations végétales. Il s'agit surtout de l'agriculture et de l'exploitation forestière pratiquée majoritairement par les populations riveraines. Cette dynamique régressive a changé la physionomie climacique des formations végétales. Les savanes arborées et arbustives constituent actuellement les formations végétales dominantes à la place des forêts claires et des forêts galeries. Les changements spatio-temporels de l'occupation du sol ont donc des effets sur la structure et la diversité spécifique des formations végétales. 


\section{References:}

1. ADEYEMI C. Pression rurale sur la forêt classée de Wari-Maro : cas du secteur allant de la latitude d'Alafiarou à celle de Bétérou. Mémoire de maîtrise de géographie. FLASH/UAC, 88 p, 2002.

2. AKOEGNINOU A. et AKPAGANA K. Etude cartographique et dynamique de la végétation de l'aire classée de la colline de Savalou (Bénin), J. Bot. SOC. BOT. Fr 3 : 69-81, 1997.

3. AROUNA O. L'exploitation des ressources biologiques et la dynamique de la forêt classée de l'Alibori Supérieur (Secteur de l'arrondissement de Bagou). Mémoire de maîtrise de géographie. FLASH/UAC, Bénin, 114 p, 2002.

4. BENOIT M . Dynamique des parcours pastoraux dans la région du Parc National du W du Niger. Niamey : Orstom, 7 p, 1998.

5. BRAUN-BLANQUET J. Plant sociology. The study of plants communities. Ed. Mc Gray Hill, New York, London, 439 p, 1932.

6. CONVERS A, CHAIBOU I, BINOT A. et DULIEU D., 2007. « La gestion de la transhumance dans la zone d'influence du parc régional du w par le programme ecopas », Vertigo - la revue électronique en sciences de l'environnement, Hors-série 4 | novembre 2007, [En ligne], mis en ligne le 27 mai 2008. URL : http://vertigo.revues.org/761. Consulté le 11 août 2010

7. FAO. Evaluation des ressources forestières mondiale. Rapport principal. 377 p, 2010.

8. FOURNIER A, YONI M. et ZOMBRE P. Les jachères à Andropogon gayanus en savane soudanienne dans l'ouest du Burkina Faso : flore, structure, déterminants et fonction dans 1'écosystème. Etudes flor. Vég. Burkina Faso, 5 : 3-32, 2002.

9. HAHN-HADJALI K.. Les groupements végétaux des savanes du sudest du Burkina Faso (Afrique de l'Ouest). Etudes flor. Vég. Burkina Faso, 3: 3-79, 1998.

10. HANSEN M, DE FRIES R, TOWSHEND J R G. et SOHLBERG R.. Global land cover classification at $1 \mathrm{~km}$ resolution using a decision tree classifier. International Journal of Remote Sensing, 21: 13311365, 2000.

11. HOUDAGBA C J, TENTE B. et GUEDOU R. Dynamique des forêts classées dans le cours moyen du fleuve Ouémé (Kétou, Dogo et Ouémé-Boukou) au Bénin. In Quelles aires protégées pour l'Afrique 
de l'Ouest? Conservation de la biodiversité et développement. Anne Fournier, Brice Sinsin et Guy Apollinaire Mensah (Editeurs). IRD Edition, collection Colloques et Séminaires, Paris, pp 368-380, 2007.

12. LE BARBE L, ALE G, MILLET B, TEXIER H. et BOREL Y. Les ressources en eau superficielles de la République du Bénin. IRD/DH, Cotonou, 540 p, 1993.

13. OUMOROU M . Etude phytosociologique de quelques phytocénoses du domaine soudanien du Bénin. Mémoire de D.E.S. en Sciences de la Terre et de la Vie, orientation Sciences Naturelles Appliquée et Ecodéveloppement. Faculté des Sciences, Université de Liège (Belgique), $82 \mathrm{p}, 1998$.

14. SALIOU A. R. A. Mode occupation des terres dans le village de Boiffo et relation avec la conservation de la zone cynégétique de la Djona. Mémoire de maîtrise de géographie. FLASH/UAC, 98 p, 2001.

15. SARR S M., 2010. Impacts des pressions anthropiques sur les ressources naturelles du parc national des "deux balé"/Burkina faso. Mémoire online. [En ligne], URL : http://www.memoireonline.com/recherche.html, consulté le 21 décembre 2010.

16. SINSIN B. Individualisation et hiérarchisation des phytocénoses soudaniennes du nord-Bénin. Bel. Journ. Bot. 127 (1) : 87-103, 1994.

17. SINSIN B., 2001. Formes de vie et diversité spécifique des associations de forêts claires du nord Bénin. Syst. Geogr. Pl. 71: 873888, 2001. 